\title{
Occult alveolar haemorrhage in pulmonary veno-occlusive disease
}

\author{
A. Rabiller*, X. Jaïs*, A. Hamid*, A. Resten\#, F. Parent*, R. Haque*, F. Capron ${ }^{\star}$, \\ O. Sitbon*, G. Simonneau* and M. Humbert*
}

ABSTRACT: Pulmonary veno-occlusive disease (PVOD) is a rare cause of pulmonary arterial hypertension that affects predominantly post-capillary pulmonary vessels. A major concern with PVOD is the poor response to available therapies and the risk of pulmonary oedema with continuous intravenous epoprostenol.

The present authors hypothesised that alveolar haemorrhage may be a characteristic feature of pulmonary veno-occlusive disease, as compared with other forms of pulmonary arterial hypertension that predominantly involve pre-capillary pulmonary arteries.

This paper reports a series of 19 patients with either PVOD $(n=8)$ or idiopathic pulmonary arterial hypertension (IPAH; $\mathbf{n}=11$ ) who underwent bronchoalveolar lavage. Cytological analyses were performed and differential counts were made on Perls-stained preparations. The Golde score was used to assess alveolar haemorrhage. As compared with IPAH, PVOD was characterised by a higher percentage of haemosiderin-laden macrophages (40 \pm 37 versus $3 \pm 6 \%$ ), resulting in elevated Golde scores ( $81 \pm 88$ versus $4 \pm 10)$.

It was concluded that occult alveolar haemorrhage is a common feature of pulmonary venoocclusive disease. Detecting occult alveolar haemorrhage may be of interest in the diagnostic approach of pulmonary veno-occlusive disease.

KEYWORDS: Alveolar haemorrhage, bronchoalveolar lavage, pulmonary arterial hypertension, pulmonary veno-occlusive disease

I diopathic pulmonary arterial hypertension (IPAH) is a disease of the small pulmonary arteries that leads to progressive increase in pulmonary vascular resistance, ultimately causing right ventricular failure and death [1, 2]. Pulmonary veno-occlusive disease (PVOD) is an uncommon cause of pulmonary arterial hypertension that affects predominantly post-capillary pulmonary vessels [3-6]. The pathological hallmark of PVOD is the extensive and diffuse occlusion of pulmonary veins by fibrous tissue [3-6]. Intimal thickening involves venules and small veins in lobular septa and, rarely, larger veins [3-6]. Clinically, patients suffering from PVOD frequently present in a similar fashion to other forms of pulmonary arterial hypertension, and PVOD accounts for $5-10 \%$ of histological forms of cases initially thought to be "idiopathic" [7].

One of the main concerns with PVOD is the poor response to available therapies and the risk of pulmonary oedema with continuous intravenous epoprostenol [8, 9]. It is well accepted that a definite diagnosis of PVOD requires histological analysis of a lung sample [6]. However, surgical lung biopsy is too invasive for these frail patients, emphasising the importance of developing less invasive tools to obtain the diagnosis [10]. The current authors, along with others, have previously shown that the diagnosis of PVOD could be suspected by analysis of clinical and radiological information, including high-resolution computed tomography (HRCT) of the chest showing a characteristic pattern combining centrilobular ground-glass opacities, septal lines and lymph nodes enlargement [11-13].

As PVOD affects the post-capillary vasculature, it may be responsible for elevated pulmonary capillary pressure and, by inference, occult alveolar haemorrhage. It was, therefore, hypothesised that alveolar haemorrhage may be a characteristic feature of PVOD, as compared with other forms of pulmonary arterial hypertension, such as IPAH affecting predominantly precapillary pulmonary arteries. The aim of this study was to evaluate whether occult alveolar haemorrhage was a characteristic feature of PVOD, as compared with IPAH.
AFFILIATIONS

${ }^{*}$ Centre des Maladies Vasculaires

Pulmonaires, Service de

Pneumologie

${ }^{\text {\#}}$ Service de Radiologie, and

'Service d'Anatomie Pathologique Unité Propre de Recherche de

l'Enseignement Supérieur EA2705, Hôpital Antoine Béclère, Université Paris-Sud, Clamart, France.

CORRESPONDENCE

M. Humbert

Service de Pneumologie

Hôpital Antoine Béclère

157, Rue de la Porte de Trivaux

92140 Clamart

France

Fax: 33146303824

E-mail: marc.humbert@abc.aphp.fr

Received:

May 062005

Accepted after revision:

August 232005

\section{SUPPORT STATEMENT}

This study was supported by grants from Legs Poix, Université Paris-Sud, Association Française contre les Myopathies, Institut National de la Santé et de la Recherche Médicale and Institut des Maladies Rares. 


\section{METHODS \\ Patients}

A total of 19 cases of "primary" pulmonary arterial hypertension, referred to Hôpital Antoine Béclère (Clamart, France) between January 1997 and May 2001, and in whom a bronchoalveolar lavage (BAL) had been performed, were retrospectively studied. Inclusion criteria were a mean pulmonary artery pressure $>3.325 \mathrm{kPa}(25 \mathrm{mmHg})$ and a pulmonary arterial wedge pressure (PAWP) $<1.995 \mathrm{kPa}$ $(15 \mathrm{mmHg})$. Causes of pulmonary hypertension such as chronic thrombo-embolic disease, chronic obstructive or restrictive lung diseases, left-heart disease, congenital heart disease, connective tissue disorders, portal hypertension and human immunodeficiency virus infection were excluded at the time of diagnosis [14]. Pulmonary function tests, arterial blood gases, carbon monoxide diffusion capacity, HRCT of the chest, right-heart catheterisation, and acute vasodilator challenge were performed in all cases $[12,15]$. In the study period, all patients with a histological confirmation of PVOD in whom BAL data were available $(n=8)$ were included. Pathological confirmation of PVOD was obtained by means of post mortem $(n=4)$, explanted lungs $(n=2)$, or open lung biopsies $(n=2)$. All IPAH patients in whom a BAL had been performed during the study period were also included $(n=11)$. The diagnosis of IPAH was established according to published guidelines on the basis of clinical, biological and haemodynamical analysis. In addition, this diagnosis was further supported by the absence of radiological abnormalities suggestive of PVOD (i.e. the absence of centrilobular pattern of ground-glass opacities, septal lines and enlarged lymph nodes on HRCT of the chest) and the absence of pulmonary oedema after initiation of chronic vasodilator therapy [7-9]. A histological confirmation of plexiform pulmonary arteriopathy was obtained in four patients (two post mortem and two explanted lungs). In the remaining seven patients, the diagnosis of IPAH did not require any invasive procedure to obtain pathological confirmation $[7,10]$.

\section{Bronchoalveolar lavage}

Fibreoptic bronchoscopy with BAL [16] was proposed to patients with PVOD because of abnormal radiological findings compatible with this diagnosis. In IPAH, it was performed either to explore a pulmonary opacity $(n=2)$ or before initiation of continuous intravenous epoprostenol $(n=9)$, after informed consent and ethics committee approval, in the context of a study, as previously described [17]. Lidocaine was used for local anaesthesia. Three $50-\mathrm{mL}$ aliquots of saline were instilled in the right middle lobe or in the subsegment that appeared to be pathological on a chest radiograph. Bacteriological and cytological analyses were performed using Gram and Papanicolaou stainings, respectively. Differential cell counts were performed on Perls-stained preparations. The Golde score was used to assess alveolar haemorrhage [18]; 200-300 macrophages were counted and each cell was graded for haemosiderin on a scale of $0-4$. A mean score for 100 cells was calculated, 0 being the minimum and 400 the maximum score [18]. Haemosiderenic resorption was considered as normal if the Golde score ranged from 0-20, medium from 20-70 and high $>70$ [18]. The Golde score was obtained in a blinded fashion in all cases.

\section{Statistical analysis}

Statistical analysis was performed using nonparametric MannWhitney U-test and the Chi-squared test. Data were presented as mean $\pm S D$. A p-value of $<0.05$ was considered as significant.

\section{RESULTS}

\section{Clinical, functional and haemodynamic information}

There was a nonsignificant male predominance in PVOD and a female predominance in IPAH (male to female ratio of 1.7:1 and $0.4: 1$, respectively; tables $1-3$ ). Moreover, there was a nonsignificant predominance of tobacco smokers in the PVOD patients (75 versus 36\%). Dyspnoea was the main symptom in all patients, and most of them were New York Heart Association functional class III or IV. The 6-min walk distance was $216 \pm 138 \mathrm{~m}$ in PVOD versus $372 \pm 82 \mathrm{~m}$ in IPAH $(\mathrm{p}=0.02)$. PVOD was associated with a more severe hypoxaemia and a more pronounced reduction of carbon monoxide diffusing capacity $(D \mathrm{~L}, \mathrm{CO})$ as compared with IPAH $(\mathrm{p}=0.007$ and $p=0.008$, respectively; table 1 ). Haemodynamics demonstrated severe pre-capillary pulmonary hypertension in all patients.

\section{HRCT of the chest}

HRCT of the chest was normal in nine of the IPAH patients. In the remaining two IPAH patients, there was a mild panlobular ground-glass opacity, which led to the indication of fibreoptic bronchoscopy. All of the PVOD patients had abnormal HRCT of the chest, showing the presence of ground-glass opacities (particularly with a centrilobular distribution), septal lines, and adenopathy (table 2). One patient had these three major criteria, six patients had two criteria and one patient had only one of the criteria. Septal lines and enlarged lymph nodes were the most frequently present signs.

\section{Bronchoalveolar lavage}

There was no evidence of infection and BAL fluids were carcinomatous cells free. As compared with IPAH, PVOD was characterised by a nonsignificant trend for elevated alveolar cell counts $\left(410,250 \pm 366,500\right.$ cells $\cdot \mathrm{mL}^{-1}$ versus $204,300 \pm 158,200$ cells $\left.\cdot \mathrm{mL}^{-1}\right)$, similar percentages of lymphocytes, neutrophils, and macrophages, but significantly higher percentages of haemosiderin-laden macrophages $(40 \pm 37$ versus $3 \pm 6 \%$; $p=0.004$; tables $2-3$ ). Occult alveolar haemorrhage was better evaluated with the Golde score, which was markedly elevated in PVOD ( $81 \pm 88$ versus $4 \pm 10 ; \mathrm{p}=0.002$; fig. 1). Only one PVOD patient had a Golde score $<20$ (fig. 1).

\section{DISCUSSION}

PVOD is an uncommon cause of pulmonary hypertension, representing $<10 \%$ of all pulmonary arterial hypertension cases, according to published series [3-6]. In the most recent classification of pulmonary hypertension, PVOD was regarded as a subset of pulmonary arterial hypertension for several reasons [7]. First, although showing predominant venous involvement, PVOD is characterised by histological changes of the small pulmonary arteries mimicking those of pulmonary arterial hypertension, including intimal fibrosis and medial hypertrophy [6, 7]. Secondly, risk factors for PVOD and pulmonary arterial hypertension are similar, including systemic scleroderma, HIV infection, and appetite suppressant exposure $[6,7,19-21]$. Thirdly, germline mutations of the gene encoding bone morphogenetic protein receptor II have been 


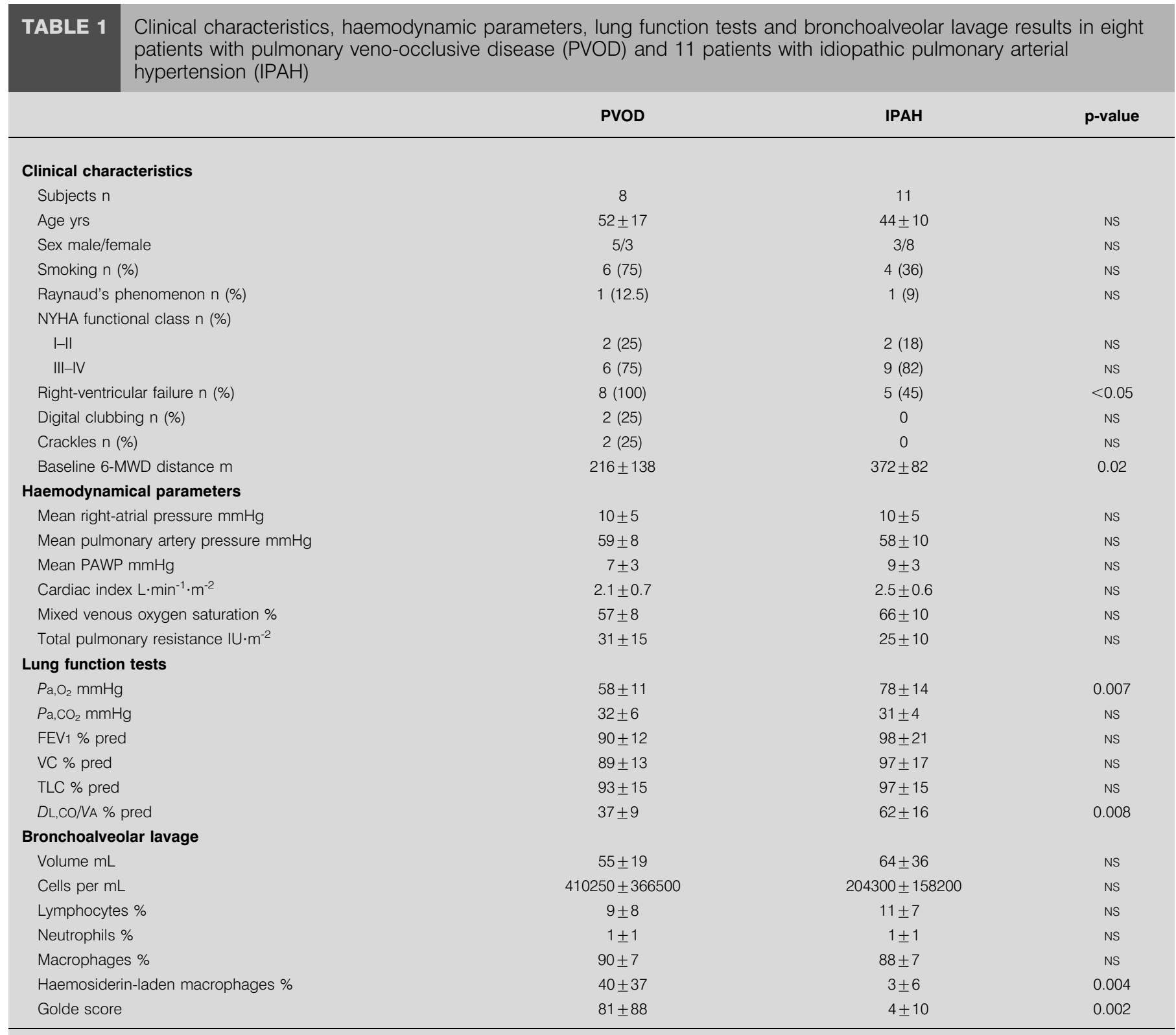

Data are presented as mean \pm SD, unless otherwise stated. NYHA: New York Heart Association; 6-MWD: 6-min walk distance; PAWP: pulmonary arterial wedge pressure; $\mathrm{Pa}_{\mathrm{O}} \mathrm{O}_{2}$ : arterial oxygen tension; $\mathrm{Pa}_{1} \mathrm{CO}_{2}$ : arterial carbon dioxide tension; $\mathrm{FEV}$ : forced expiratory volume in one second; \% pred: percentage predicted; $\mathrm{VC}$ : vital capacity; TLC: total lung capacity; DL,CO/NA: carbon monoxide diffusing capacity/alveolar volume; NS: nonsignificant. $1 \mathrm{mmHg}=0.133 \mathrm{kPa}$.

recognised in patients with familial pulmonary arterial hypertension and IPAH, as well as in cases of PVOD [22]. Supporting the concept that these two conditions may overlap, there are reports of patients displaying PVOD or IPAH in the same families [7]. Fourthly, clinical presentation of patients with PVOD is often undistinguishable from that of patients with IPAH [13].

According to recent guidelines, PVOD requires similar clinical management as other forms of pulmonary arterial hypertension [7]. However, prognosis seems to be worse in this subset of patients, with a more rapid downhill course [7]. In addition, vasodilators and especially continuous intravenous epoprostenol therapy have to be used with great caution because of the risk of severe pulmonary oedema $[8,9]$. As a result, it is critical to establish this diagnosis as early as possible, in order to consider lung transplantation for eligible patients [7-9].

More than 200 cases of PVOD have been reported in the literature. It affects all age groups without any geographical predilection. A male predominance has been shown in PVOD, as opposed to what is found in IPAH [13]. The cause of PVOD remains obscure, but the process may be due to multiple factors that provoke a common pattern of vascular injury and repair [23]. Some authors have proposed a viral aetiology, including infection with the HIV [21, 24]. Haematological malignancies, as well as chemotherapy (bleomycin, gemcitabin, etc.), radiotherapy or bone marrow transplantation are 


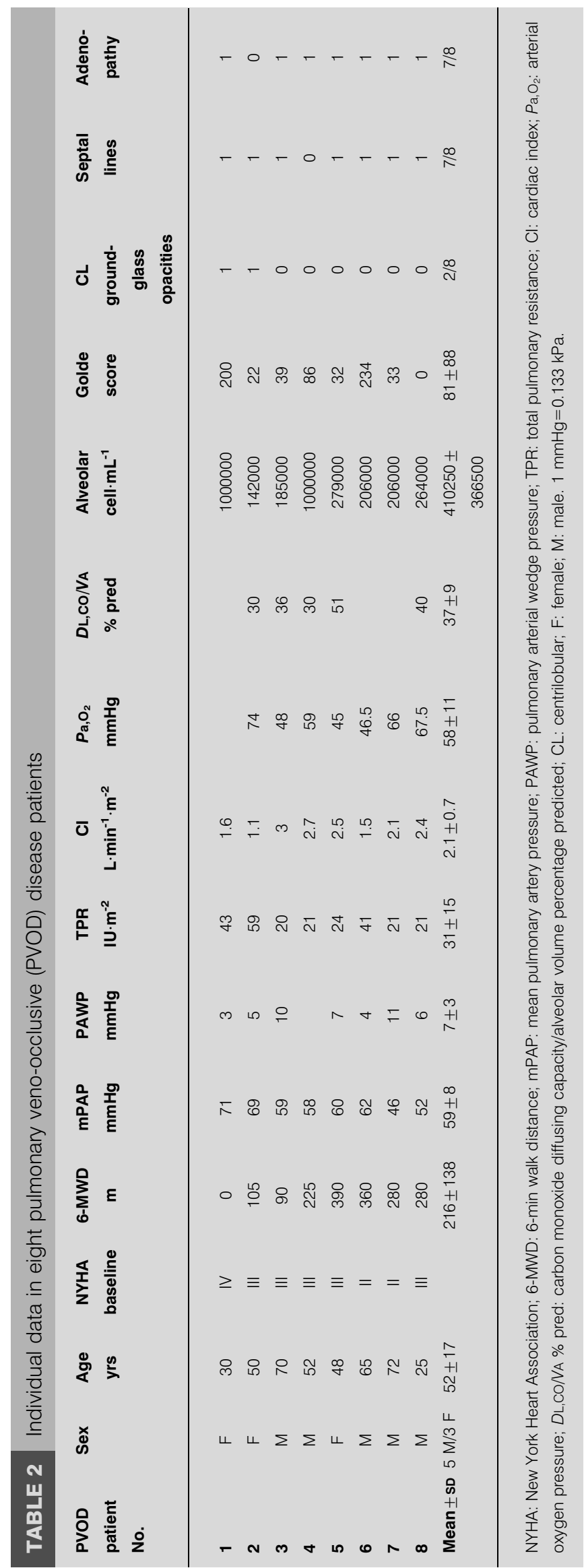

other potential causes of PVOD [25-30]. In this study, the current authors found a nonsignificant predominance of tobacco smokers in PVOD patients. It has been speculated that a toxic exposure could be responsible for occult alveolar haemorrhage and impaired function of alveolar macrophages: increased percentages of haemosiderin-laden alveolar macrophages were found in BAL fluid of cocaine smokers [31] and an impairment in nitric oxide production was observed in alveolar macrophages from smokers of marijuana and cocaine $[32,33]$. This chronic damage of the alveoli and of pulmonary microcirculation is not clear as far as tobacco exposure is concerned [31].

A definite diagnosis of PVOD is difficult to establish in the living patients. Indeed, examination of a surgical lung biopsy is necessary to obtain a histological confirmation, but this invasive procedure is too hazardous in patients with a major haemodynamic compromise [10]. Therefore, less invasive methods to approach this diagnosis are now proposed in order to obtain a high index of suspicion [12].

Clinical presentation of these patients is often similar to that of patients with IPAH [13]. However, findings suggestive of a diagnosis other than IPAH may be identified in PVOD, such as digital clubbing and bibasal crackles [13]. Despite reports of elevated PAWP in cases of PVOD, haemodynamical parameters are, in fact, usually identical to that of IPAH with a normal PAWP despite post-capillary involvement [23, 34]. This paradoxical normal PAWP is due to the fact that the disease process occurs in the small pulmonary septal veins with little or no obstruction of the larger pulmonary veins $[4,6,25]$. Therefore, when PAWP is measured during right-heart catheterisation, the static column of blood produced is unaffected by the occlusion and reflects the normal pressure in the larger veins, not the elevated pressure in the capillaries $[34,35]$.

As compared with IPAH, the present data suggest that PVOD is associated with a more pronounced hypoxaemia and a severe reduction of $\mathrm{DL}, \mathrm{CO}$, while spirometry and lung volume measurements are within normal limits [36]. These abnormalities may be explained by interstitial oedema and/or pulmonary capillary angiogenesis secondary to chronic pulmonary venous obstruction [37]. Interstitial lung disease may indeed be identified in PVOD [12]. HRCT of the chest allows a better analysis, and the current data confirm the results of previous studies, which have emphasised the relevance of this technique in the noninvasive approach of PVOD [12]. Centrilobular pattern of ground-glass opacities, septal lines and enlarged lymph nodes are the main abnormal findings suggestive of PVOD [12]. These abnormalities may be present on baseline HRCT of the chest and strongly correlate with a risk of lifethreatening pulmonary oedema with continuous intravenous epoprostenol therapy [11, 12].

As PVOD mainly affects the post-capillary pulmonary vessels, it was hypothesised that it may be responsible for chronic occult alveolar haemorrhage. Therefore, cells harvested by BAL in patients with PVOD or IPAH were studied. As compared to IPAH patients, patients displaying PVOD indeed had a higher percentage of haemosiderin-laden macrophages and an elevated Golde score [18], confirming occult alveolar haemorrhage in most cases of PVOD. 


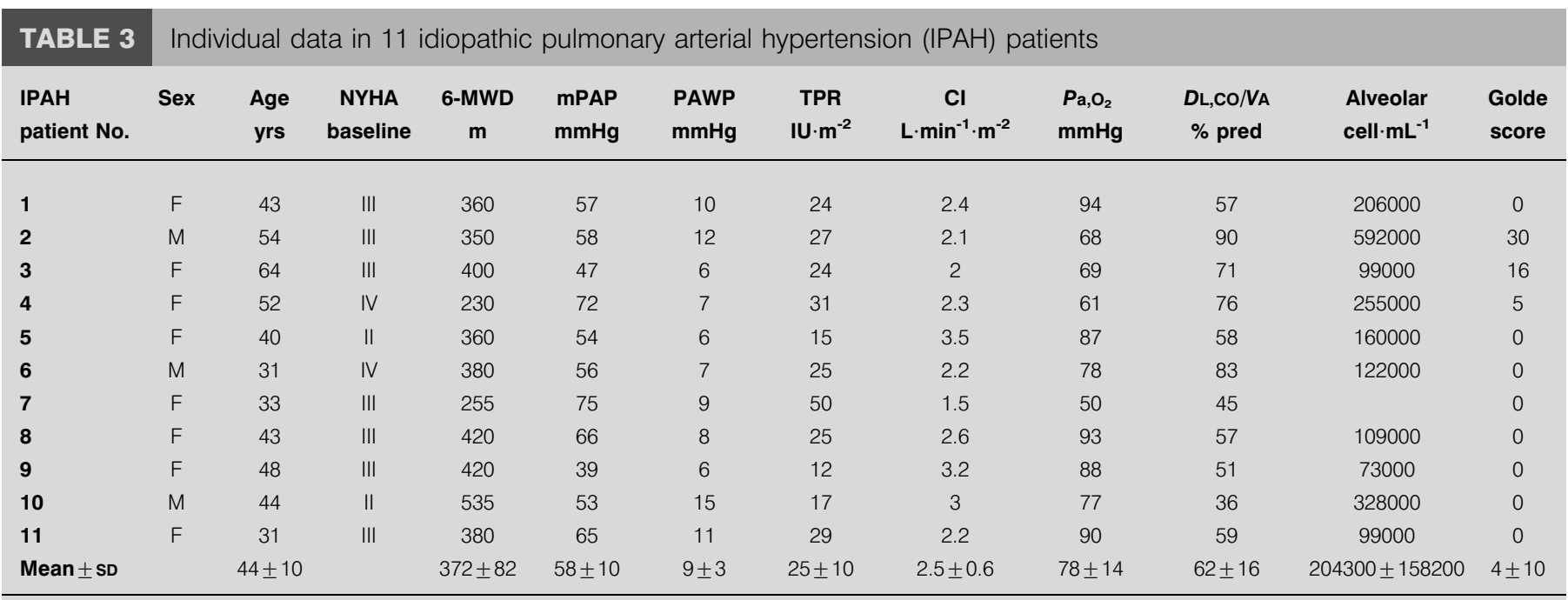

NYHA: New York Heart Association; 6-MWD: 6-min walk distance; mPAP: mean pulmonary artery pressure; PAWP: pulmonary artery wedge pressure; TPR: total pulmonary resistance; $\mathrm{Cl}$ : cardiac index; $\mathrm{Pa}, \mathrm{O}_{2}$ : arterial oxygen pressure; $\mathrm{DL}, \mathrm{CO} / \mathrm{VA} \%$ pred: carbon monoxide diffusing capacity/alveolar volume percentage predicted; $\mathrm{F}$ : female; M: male. $1 \mathrm{mmHg}=0.133 \mathrm{kPa}$.

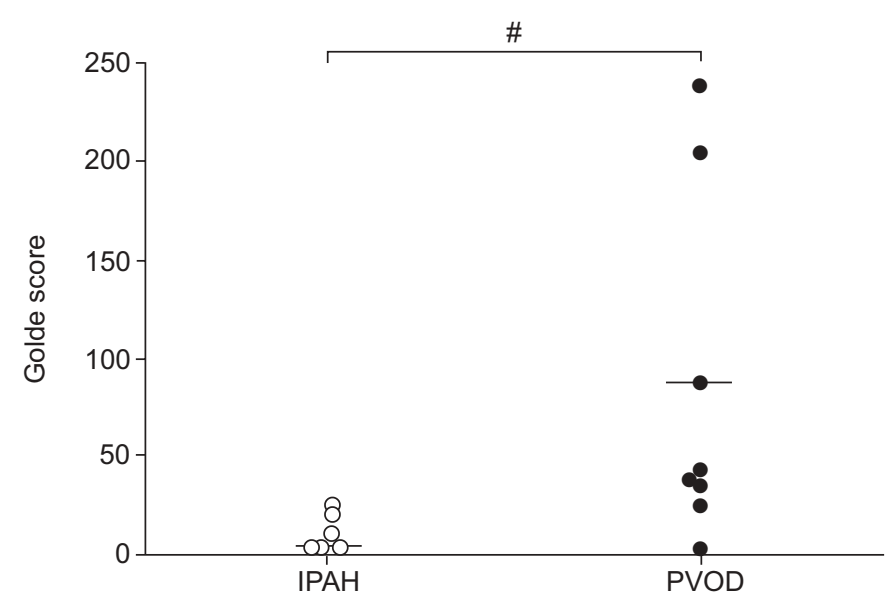

FIGURE 1. Golde score in patients with idiopathic pulmonary arterial hypertension (IPAH) or pulmonary veno-occlusive disease (PVOD). ${ }^{*}: p=0.002$.

This case series has several limitations. The analysis was retrospective. However, conducting a prospective trial would be extremely difficult in such a rare disorder and a majority of patients would not have a definite diagnosis of PVOD because lung biopsy is not recommended in these unstable patients. Lung transplantation or post mortem analysis of the lungs is available in only a minority of patients in any cohorts followed in pulmonary vascular centres. Moreover, BAL is not usually performed in patients with IPAH, unless there is some suspicion of associated pulmonary parenchymal disease. The current authors, therefore, proposed to use the results of a series of BAL that are available at their institution, as a comparator for this study [17]. A histological confirmation was obtained in all eight PVOD patients and in four of the 11 IPAH patients. According to recent guidelines [7], the diagnosis of
IPAH does not require any pathological confirmation. Indeed, examination of a surgical lung biopsy would be necessary to obtain a histological confirmation, but this invasive procedure is too hazardous in patients with a major haemodynamic compromise [10]. Thus, a diagnosis of IPAH was established in all 11 patients on the basis of recently published guidelines [7], and was confirmed by the absence of radiological abnormalities suggestive of PVOD and the absence of vasodilatorinduced pulmonary oedema.

In conclusion, the current study indicates that occult alveolar haemorrhage is a common feature of pulmonary venoocclusive disease. The present authors propose that patients with a clinical suspicion of pulmonary veno-occlusive disease should benefit from a less invasive approach combining high-resolution computed tomography of the chest and bronchoalveolar lavage analysis to detect occult alveolar haemorrhage.

\section{REFERENCES}

1 Rubin LJ. Primary pulmonary hypertension. N Engl J Med 1997; 336: 111-117.

2 Runo JR, Loyd JE. Primary pulmonary hypertension. Lancet 2003; 361: 1533-1544.

3 Heath D, Segel N, Bishop J. Pulmonary veno-occlusive disease. Circulation 1966; 34: 242-248.

4 Wagenvoort CA, Wagenvoort N, Takahashi T. Pulmonary veno-occlusive disease: involvment of pulmonary arteries and review of the literature. Human Pathol 1985; 16: 1033-1041.

5 Mandel J, Mark EJ, Hales CA. Pulmonary veno-occlusive disease. Am J Respir Crit Care Med 2000; 162: 1964-1973.

6 Pietra GG, Capron F, Stewart S, et al. Pathologic assessment of vasculopathies in pulmonary hypertension. J Am Coll Cardiol 2004; 43: Suppl. 12, 25S-32S. 
7 Simonneau G, Galiè N, Rubin LJ, et al. Clinical classification of pulmonary hypertension. J Am Coll Cardiol 2004; 43: Suppl. 12, 5S-12S.

8 Palmer SM, Robinson LJ, Wang A, Gossage JR, Bashore T, Tapson VF. Massive pulmonary edema and death after prostacyclin infusion in a patient with pulmonary venoocclusive disease. Chest 1998; 113: 237-240.

9 Humbert M, Sitbon O, Simonneau G. Treatment of pulmonary arterial hypertension. N Engl J Med 2004; 351: 1425-1436.

10 Nicod P, Moser KM. Primary pulmonary hypertension: the risk and benefit of lung biopsy. Circulation 1989; 80: 1486-1488.

11 Resten A, Maitre S, Humbert M, Capron F, Simonneau G, Musset D. Pulmonary arterial hypertension: thin-section CT predictors of epoprostenol therapy failure. Radiology 2002; 222: 782-788.

12 Resten A, Maitre S, Humbert M, et al. Pulmonary hypertension: CT of the chest in pulmonary veno-occlusive disease. Am J Roentgenol 2004; 183: 65-70.

13 Holcomb BW Jr, Loyd JE, Ely EW, Johnson J, Robbins IM. Pulmonary veno-occlusive disease: a case series and new observations. Chest 2000; 118: 1671-1679.

14 Rich S, Dantzker DR, Ayers SM, et al. Primary pulmonary hypertension: a national prospective study. Ann Intern Med 1987; 107: 216-223.

15 Sitbon O, Humbert M, Jagot JL, et al. Inhaled nitric oxide as a screening agent to safely identify responders to oral calcium-channel blockers in primary pulmonary hypertension. Eur Respir J 1998; 12: 265-270.

16 Reynolds HY. Bronchoalveolar lavage. Am Rev Respir Dis 1987; 135: 250-263.

17 Martin I, Humbert M, Marfaing-Koka A, et al. Plasminogen activation by blood monocytes and alveolar macrophages in primary pulmonary hypertension. Blood Coagul Fibrinolysis 2002; 13: 417-422.

18 Golde DW, Drew WL, Klein HZ, Finley TN, Cline MJ. Occult pulmonary haemorrhage in leukaemia. BMJ 1975; 2: 166-168.

19 Morassut PA, Walley VM, Smith CD. Pulmonary venoocclusive disease and the CREST variant of scleroderma. Can J Cardiol 1992; 8: 1055-1058.

20 Dorfmüller $\mathrm{P}$, Humbert $\mathrm{M}$, Sanchez $\mathrm{O}$, Sitbon O, Simonneau G, Capron F. Significant occlusive lesions of pulmonary veins are common in patients with pulmonary hypertension associated with connective tissue disease. Am J Respir Crit Care Med 2002; 167: A694.

21 Escamilla R, Hermant C, Berjaud J, Mazerolles C, Daussy X. Pulmonary veno-occlusive disease in a HIV-infected intravenous drug abuser. Eur Respir J 1995; 8: 1982-1984.

22 Runo JR, Vnencak-Jones CL, Prince M, et al. Pulmonary veno-occlusive disease caused by an inherited mutation in bone morphogenetic protein receptor II. Am J Respir Crit Care Med 2003; 167: 889-894.

23 Case records of the Massachusetts General Hospital. N Engl J Med 1993; 2: 1720-1728.

24 McDonnell PJ, Summer WR, Hutchins GM. Pulmonary veno-occlusive disease. Morphological changes suggesting a viral cause. JAMA 1981; 246: 667-671.

25 Lombard CM, Churg A, Winokur S. Pulmonary venoocclusive disease following therapy for malignant neoplasms. Chest 1987; 92: 871-876.

26 Vansteenkiste JF, Bomans P, Verbeken EK, Nackaerts KL, Demedts MG. Fatal pulmonary veno-occlusive disease possibly related to gemcitabine. Lung Cancer 2001; 31: 83-85.

27 Gagnadoux F, Capron F, Lebeau B. Pulmonary venoocclusive disease after neoadjuvant mitomycin chemotherapy and surgery for lung carcinoma. Lung Cancer 2002; 36: 213-215.

28 Knight BK, Rose AG. Pulmonary veno-occlusive disease after chemotherapy. Thorax 1985; 40: 874-875.

29 Williams LM, Fussell S, Veith RW, Nelson S, Mason CM. Pulmonary veno-occlusive disease in an adult following bone marrow transplantation. Chest 1996; 109: 1388-1391.

30 Kramer MR, Estenne M, Berkman N, et al. Radiationinduced pulmonary veno-occlusive disease. Chest 1993; 104: 1282-1284.

31 Baldwin GC, Choi R, Roth MD, et al. Evidence of chronic damage to the pulmonary microcirculation in habitual users of alkaloidal ("crack") cocaine. Chest 2002; 121: 1231-1238.

32 Roth MD, Whittaker K, Salehi K, Tashkin DP, Baldwin GC. Mechanisms for impaired effector function in alveolar macrophages from marijuana and cocaine smokers. J Neuroimmunol 2004; 147: 82-86.

33 Shay $\mathrm{AH}$, Choi $\mathrm{R}$, Whittaker $\mathrm{K}$, et al. Impairment of antimicrobial activity and nitric oxide production in alveolar macrophages from smokers of marijuana and cocaine. J Infect Dis 2003; 187: 700-704.

34 Weed HG. Pulmonary "capillary" wedge pressure not the pressure in the pulmonary capillaries. Chest 1991; 100: 1138-1140.

35 Fesler P, Pagnamenta A, Vachiery JL, et al. Single arterial occlusion to locate resistance in patients with pulmonary hypertension. Eur Respir J 2003; 21: 31-36.

36 Elliott CG, Colby TV, Hill T, Crapo RO. Pulmonary venoocclusive disease associated with severe reduction singlebreath carbon monoxide diffusion capacity. Respiration 1988; 53: 262-266.

37 Schraufnagel GE, Sekosan M, McGee T, Thakkar MB. Human alveolar capillaries undergo angiogenesis in pulmonary veno-occlusive disease. Eur Respir J 1996; 9: 346-350. 